\title{
Síndrome de apnea obstructiva del sueño persistente en niños adenoamigdalectomizados: artículo de revisión
}

\author{
Persistent obstructive sleep apnea syndrome in adenotonsillectomized \\ children: review article
}

Francisco Ringler A. ${ }^{1}$, Pilar Gajardo O., ${ }^{2,3,4}$

\begin{abstract}
Resumen
El síndrome de apnea e hipoapnea obstructiva del sueño (SAHOS) en niños forma parte del espectro de trastornos respiratorios del sueño en la infancia. Tiene una prevalencia entre un $0,69 \%$ y $4,7 \%$ en la población infantil con una mayor incidencia entre los 2 y 6 años debido principalmente a la hiperplasia adenoamigdaliana y constituye una importante causa de morbilidad neurocognitiva y conductual en quienes lo padecen. El principal tratamiento quirúrgico del SAHOS infantil lo constituye la adenoamigdalectomía, cirugía que logra la resolución de los síntomas entre un $20 \%$ y $75 \%$ de los pacientes. Los pacientes con comorbilidades asociadas tales como obesidad, enfermedades neuromusculares y alteraciones craneofaciales, entre otras, tienen mayor riesgo de SAHOS persistente. En la presente revisión de la literatura abordaremos el diagnóstico, enfrentamiento, estudio y tratamiento del SAHOS persistente posadenoamigdalectomía en niños.

Palabras clave: apnea obstructiva del sueño, niños, adenoamigdalectomía, persistente, tratamiento.
\end{abstract}

\begin{abstract}
Obstructive sleep apnea syndrome (OSA) in children is part of the spectrum of respiratory sleep disorders in childhood. It has a prevalence between 0.69 and $4.7 \%$ in pediatric population, with a higher incidence between 2 and 6 years old, mainly due to adenotonsillar hyperplasia. OSA constitutes an important cause of neurocognitive and behavioral morbidity. The main surgical treatment for childhood OSA is adenotonsillectomy, which resolves symptoms in 20\%-75\% of patients. Patients with associated comorbidities such as obesity, neuromuscular diseases, and craniofacial malformations are at higher risk of having persistent OSA. In this literature review, we will discuss the diagnosis, evaluation and treatment of persistent OSA after adenotonsillectomy in children.
\end{abstract}

Keywords: obstructive sleep apnea syndrome, pediatrics, adenotonsillectomy, persistent, treatment.

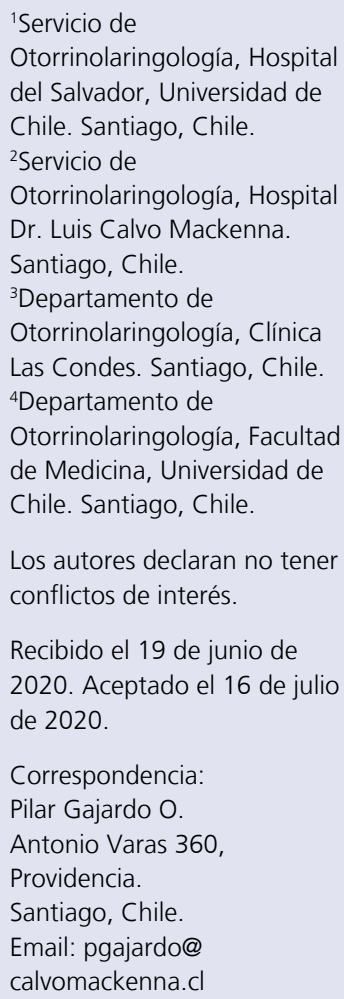

\section{Introducción}

Los trastornos respiratorios del sueño (TRS) incluyen un amplio espectro de entidades, que van desde el roncador primario (RP), el síndrome de resistencia de la vía aérea superior (SRVAS) y el síndrome de apnea e hipoapnea obstructiva del sueño (SAHOS $)^{1}$. El SAHOS infantil se define como una disfunción de la vía aérea superior (VAS) durante el sueño, caracterizado por eventos recurrentes de obstrucción parcial o completa de la VAS con alteración de la oxigenación normal, ventilación y patrones de sueño ${ }^{2}$. Su patogenia corresponde a un proceso dinámico donde confluyen tanto factores anatómicos como funcionales que generan un desequilibrio y colapso de la VAS ${ }^{3}$. La prevalencia estimada de SAHOS infantil a nivel mundial es de $0,69 \%$ a $4,7 \%{ }^{4}$ con una incidencia mayor entre los 2 a 6 años que se relaciona con el aumento del tejido adenotonsilar, y un segundo peak de incidencia 
entre los 12 a 15 años relacionado con el aumento de peso y la obesidad ${ }^{1}$. En Chile existen limitados estudios epidemiológicos de SAHOS en población pediátrica. Sánchez y cols. ${ }^{5}$ realizaron una estimación de la prevalencia de TRS en escolares de $1^{\circ}$ básico mediante cuestionario de sueño pediátrico validado al español (pediatric sleep questionnaire, $\mathrm{PSQ}^{6}$ ) y determinaron que un $17,7 \%$ sufre de TRS, comparable con las tasas más altas a nivel mundial.

\section{Manifestaciones clínicas y sus consecuencias}

Las manifestaciones clínicas del SAHOS infantil las podemos dividir en síntomas diurnos y nocturnos como se aprecia en la Tabla $1^{7}$.

Las consecuencias inmediatas de la obstrucción de la VAS durante el sueño generan aumento del esfuerzo respiratorio, hipoxemia intermitente, fragmentación del sueño e hipoventilación alveolar, que desencadenan un aumento del stress oxidativo, activación de la cascada inflamatoria sistémica, disfunción endotelial y activación simpática, responsables de los efectos y consecuencias a mediano y largo plazo del SAHOS a nivel neurocognitivo, metabólico y cardiovascular ${ }^{3,7}$. Numerosos estudios informan que el SAHOS está asociado con una disminución de las puntuaciones neurocognitivas, conductuales y de calidad de vida, así como con un aumento de la presión arterial sistémica, secuelas pulmonares aumentadas y un mayor uso de atención médica.

\section{Diagnóstico del SAHOS}

La evaluación de los niños con SAHOS debe incluir la historia clínica y examen físico con especial atención en el peso corporal, tamaño amigdalino, anatomía nasal, micro/ retrognatia, forma del paladar, maloclusión dental y otras alteraciones craneofaciales ${ }^{8,9}$. Esta evaluación a menudo debe incluir la nasofibrolaringoscopía (NFC) flexible para examinar la cavidad nasal, determinar el tamaño adenoídeo y del polo inferior de las amígdalas, observar la base de lengua y la anatomía laríngea, con el fin de determinar los niveles de obstrucción de la VAS. Aunque esta evaluación es importante, la historia y el examen físico por sí solos son insuficientes para diagnosticar el SAHOS en niños ${ }^{8,9}$. El gold standard para el diagnóstico del SAHOS infantil es la polisomnografía nocturna en un laboratorio de sueño ${ }^{8,10}$ que evalúa parámetros neurofisiológicos y cardiorrespiratorios. Según el índice de apneas/hipoapneas (IAH: número de apneas/hipoapneas por hora de sueño) clasificamos el SAHOS en leve, moderado y severo como se observa en la Tabla $2^{8}$.

La poligrafía respiratoria (PR) es un examen de menor costo y mayor accesibilidad que consta de al menos 4 canales que evalúan solo variables respiratorias: flujo aéreo, pulsioximetría y esfuerzo respiratorio. Varios estudios han evaluado la correlación entre el IAH en PSG y PR con resultados variables; sin embargo, la mayoría concluye que la $\mathrm{PR}$ es confiable en el SAHOS pediátrico moderado a severo $^{11,12}$. Por su parte, la oximetría nocturna

Tabla 1. Síntomas y signos SAHOS infantil

\begin{tabular}{ll}
\hline Nocturnos & Diurnos \\
\hline Ronquidos & Respirador bucal \\
\hline Apneas observadas & Hiperactividad \\
\hline Posturas anómalas al dormir & Déficit atencional \\
\hline Sueño inquieto & Disminución rendimiento escolar \\
\hline Terrores nocturnos, sonambulismo & Alteraciones conductuales \\
\hline Enuresis & Somnolencia diurna \\
\hline Sudoración nocturna & Cefalea matinal \\
\hline & Sequedad bucal \\
\hline
\end{tabular}


Tabla 2. Severidad del SAHOS infantil según

IAH

\begin{tabular}{cc}
\hline Severidad del SAHOS & IAH \\
Leve & 1 a 4,9 \\
Moderado & 5 a 9,9 \\
Severo & 10 o > \\
\hline
\end{tabular}

tiene un alto valor predictivo positivo (VPP) de $97 \%$ para detectar SAHOS, pero un valor predictivo negativo (VPN) de solo un $43 \%$ en comparación con la polisomnografía, siendo alta la tasa de falsos negativos con esta técnica diagnóstica, sobre todo en los casos de SAHOS leve ${ }^{13,14}$, por lo que en algunos centros es utilizada como herramienta de categorización de riesgo perioperatorio y para establecer tiempos de espera para la cirugía ${ }^{14}$.

\section{Tratamiento}

La adenoamigdalectomía (AA) es el tratamiento quirúrgico de primera línea para niños con SAHOS e hiperplasia adenoamigdalina(HAA) ${ }^{15,16}$, avalada por la American Academy of Pediatrics y la American Academy of Otolaryngology-Head and Neck Surgery ${ }^{10,17}$. Es de suma importancia tener presente que los niños con SAHOS pueden tener obstrucción de la VAS en uno o más niveles, sobre todo en pacientes con comorbilidades y, por lo tanto, la amigdalectomía y/o adenoidectomía pueden fallar como tratamiento definitivo ${ }^{18}$.

Entre el 1 de enero de 2015 y el 31 de diciembre de 2019 se realizaron 2.292 amigdalectomías c/s adenoidectomía en el hospital Dr. Luis Calvo Mackenna, siendo la principal indicación la hiperplasia amigdalina asociada a roncopatía. Respecto a las tasas de curación del SAHOS, no conocemos con certeza su valor, puesto que no existen estudios nacionales que lo evidencien. Por otra parte, es importante señalar que en nuestro país y en especial en el sistema público de salud, la disponibilidad de laboratorios de sueño para el completo estudio preoperatorio de estos pacientes es limitada, por lo que la decisión quirúrgica se basa en la historia clínica, hallazgos sugerentes al examen físico y cuestionarios tales como el PSQ. En gran parte del mundo la situación no dista demasiado de la realidad nacional. Encuestas realizadas a otorrinolaringólogos generales y pediátricos estadounidenses muestran que aproximadamente el $90 \%$ de AA por TRS se realizan sin un estudio preoperatorio del sueño ${ }^{19}$.

Los estudios de sueño pos-AA se han asociado con mejoras significativas en los principales parámetros de la arquitectura del sueño, con un número significativamente menor de despertares nocturnos y mayor eficiencia del sueño, aunque sin diferencias significativas en el porcentaje de sueño REM. Bhattacharjee y cols. ${ }^{15}$ evidenciaron una reducción significativa del IAH en un $90,1 \%$ de los niños AA, pero de los cuales solo un 27,2\% logró la curación total del SAHOS $(\mathrm{IAH}<1)$. En una revisión sistemática se evidenciaron tasas de curación del SAHOS post-AA en niños no obesos y sin comorbilidades de un $74 \%$ versus un $39 \%$ en pacientes con comorbilidades como obesidad, SAHOS severo o en menores de $3 \operatorname{años}^{20}$ y también se concluye que un $34 \%$ de los niños AA presentaron un IAH mayor o igual a 5 posterior a la cirugía. En un metaanálisis de 4 estudios y 110 niños obesos se encontró que el $51 \%$ tenía un IAH superior a 5 luego de la $\mathrm{AA}^{21}$. Actualmente la evidencia es clara y confirma que la AA por sí sola es insuficiente para el tratamiento del SAHOS en un porcentaje importante de los pacientes, lo que fluctúa entre un $20 \%$ y $75 \%$ según la presencia o no de comorbilidades asociadas y del criterio polisomnográfico adoptado para definir SAHOS residual o persistente ${ }^{15,22}$.

\section{SAHOS persistente y comorbilidades asociadas}

A la fecha no existe consenso en la literatura internacional respecto a su definición y criterios polisomnográficos del SAHOS persistente luego de la AA en niños ${ }^{15,16,22}$, pero lo que sí está claro es que la identificación temprana y el tratamiento del SAHOS persistente infantil son cruciales para prevenir sus complicaciones, para planificar el tratamiento médico y/o quirúrgico, y para determinar la necesidad de un seguimiento a largo plazo. Se han establecido como factores de riesgo para SAHOS persistente edad menor de 3 años o mayor de 7 
años, obesidad, SAHOS severo, raza afroamericana y la presencia de comorbilidades como el asma bronquial, anomalías craneofaciales y enfermedades neuromusculares, entre otros, como se muestra en la Tabla 3.

\section{Diagnóstico del SAHOS persistente}

La pregunta que surge entonces es: $i^{a}$ quiénes? ¿cuándo? y ¿cómo estudiamos a los pacientes con SAHOS persistente pos-AA? La American Academy of Sleep Medicine (AASM) recomienda la PSG como el gold standard en la evaluación de la respuesta a la AA en pacientes con SAHOS infantil ${ }^{23}$, la cual debe ser realizada al menos 6 semanas después de la $\mathrm{AA}^{15}$. Existen distintas guías clínicas que realizan sus recomendaciones respecto al estudio con PSG pre

\section{Tabla 3. Factores de riesgo SAHOS persistente pos-AA}

\begin{tabular}{|c|c|}
\hline Categoría & Factores de riesgo SAHOS persistente \\
\hline Edad & $<3$ años y > 7 años \\
\hline Peso & Obesidad (IMC z-score >1,65) \\
\hline Polisomnografía & SAHOS severo $(\mathrm{IAH}>\mathrm{O}=10)$ \\
\hline $\begin{array}{l}\text { Síndromes } \\
\text { genéticos }\end{array}$ & $\begin{array}{l}\text { Trisomía } 21 \\
\text { Síndromes craneofaciales } \\
\text { Síndrome de Prader-Willi } \\
\text { Mucopolisacaridosis }\end{array}$ \\
\hline $\begin{array}{l}\text { Alteraciones } \\
\text { neuromusculares }\end{array}$ & $\begin{array}{l}\text { Distrofia muscular } \\
\text { Malformación de Arnold Chiari }\end{array}$ \\
\hline Comorbilidades & Asma bronquial \\
\hline Raza & Afroamericana \\
\hline
\end{tabular}

y pos-AA que se muestran en la Tabla $4^{23,25}$.

Todos los pacientes con factores de riesgo para SAHOS residual, SAHOS severo preoperatorio y aquellos con persistencia de síntomas pos-AA deben ser estudiados con PSG. Sin embargo, sabemos que esta recomendación no es factible de cumplir a cabalidad en nuestra realidad nacional, por lo que al final de este artículo proponemos un algoritmo de trabajo en pacientes con SAHOS residual con el fin de optimizar los escasos recursos disponibles.

En países donde los recursos financieros o la disponibilidad de PSG son limitados, existe un interés creciente en estrategias alternativas para identificar el SAHOS persistente. Estos incluyen poligrafía respiratoria (PR), registro clínico del sueño (RCS) y estrategias basadas en biomarcadores. Durante la última década ha habido un considerable interés en la identificación de biomarcadores para la enfermedad residual ${ }^{26}$. Una revisión sistemática de biomarcadores para el diagnóstico de SAHOS identificó solo 1 estudio en niños que exhibió una precisión diagnóstica que se consideró lo suficientemente sólida para el diagnóstico potencial de SAHOS pediátrico. En este estudio se analizaron muestras de orina matinal en 60 niños con SAHOS confirmado con PSG y un grupo control de niños con roncopatía primaria. En los niños con SAHOS encontraron alteraciones específicas y consistentes en las concentraciones urinarias de ciertas proteínas (uromodulina, urocortina-3, orosomucoide-1 y calicreína), y que la combinación de 3 o 4 proteínas presentes sobre el valor normal ten-

Tabla 4. Recomendaciones guías clínicas americanas de estudio con PSG

\begin{tabular}{|c|c|c|}
\hline Guía clínica & PSG preoperatoria & PSG posoperatoria \\
\hline AAO-HNS 2011 & $\begin{array}{l}\text { Riesgo de complicaciones perioperatorias } \\
\text { Discordancia clínica }\end{array}$ & \\
\hline AAP 2012 & Ronquidos + síntomas de SAHOS & $\begin{array}{l}\text { FR SAHOS persistente } \\
\text { SAHOS severo preoperatorio } \\
\text { Persistencia de síntomas }\end{array}$ \\
\hline AASM 2012 & Sospecha clínica de SAHOS & $\begin{array}{l}\text { FR SAHOS persistente } \\
\text { SAHOS moderado o severo preoperatorio } \\
\text { Persistencia de síntomas }\end{array}$ \\
\hline
\end{tabular}

AAO-HNS: American Academy of Otolaryngology-Head and Neck Surgery. AAP: American Academy of Pediatrics. AASM: American Academy of Sleep Medicine.

FR: factores de riesgo. 
dría una sensibilidad del 95\% y especificidad de un $100 \%$ en la predicción del SAHOS ${ }^{27}$.

\section{Enfrentamiento del paciente con SAHOS persistente}

Frente a un paciente con SAHOS persistente lo primero a determinar es el o los niveles a los cuales ocurre el colapso de la VAS. Si bien, no hay consenso sobre los métodos óptimos para identificar sitios de obstrucción de la VAS en niños con enfermedad persistente pos-AA ${ }^{28}$, la endoscopía del sueño inducida por fármacos (DISE) y las imágenes de resonancia magnética cine (RM cine) son las más utilizadas. En ambas técnicas, se evalúan múltiples sitios anatómicos en forma dinámica mientras el paciente se encuentra bajo sueño inducido farmacológicamente y en ventilación espontánea intentando simular el sueño fisiológico ${ }^{18}$.

\section{DISE}

Esta técnica permite la evaluación anatómica y dinámica de la VAS durante sueño inducido por medio de una fibra óptica flexible. Su introducción en 1991 por Pringle y Croft condujo al primer método objetivo de selección de pacientes y desarrolló el concepto de tratamiento individualizado en pacientes SAHOS $^{29}$. Dos de los protocolos más utilizados internacionalmente son los del Cincinnati Children's Hospital M.C. ${ }^{30}$ y Antwerp University Hospital ${ }^{31}$ que se muestran en la Tabla 5.

Entre las diferencias farmacocinéticas y efectos adversos de los distintos anestésicos utilizados se describen las siguientes: propofol es un hipnótico no barbitúrico que actúa sobre el complejo receptor GABA-A inhibiendo la transmisión sináptica, es de vida media corta con un rápido efecto sedante (30-60 segun- dos) y una recuperación completa luego de 10 minutos desde su administración, pero tiene riesgo de hipotensión y depresión respiratoria, aunque menor al compararlo con benzodiazepinas como midazolam. Respecto a dexmedetomidina, corresponde a un estimulante de receptores alfa-2-adrenérgicos que logra rápidamente un efecto sedante y también analgésico, pero al tener una vida media más prolongada (2 horas) tiene el riesgo de sobresedación y dificultad para despertar al paciente. La principal ventaja de dexmedetomidina radica en que no genera depresión respiratoria ${ }^{31,34}$.

Se han descrito variados sistemas de descripción de DISE en niños, no existiendo en la actualidad preferencia de uno sobre otro, sin embargo, existe consenso en que en niños se debe incluir la presencia de obstrucción a nivel nasal, nasofaríngea, velo del paladar, orofaringe y laringe, junto con describir si las maniobras de reposición mandibular son capaces de mejorar la obstrucción de la VAS. El estudio de Friedmann y cols. ${ }^{35}$, evidenció que la frecuencia de DISE en instituciones pediátricas variaba entre 0,5 a 20 por mes con una baja tasa de acuerdo entre los establecimientos examinados (33\%). En cuanto al momento de la realización del DISE y la toma de decisiones quirúrgicas, la mayoría lo realiza en SAHOS persistente pos-AA y no como parte del estudio inicial. Además, al consultar sobre su uso como herramienta diagnóstica y terapéutica en el mismo tiempo quirúrgico, solo una institución lo realiza en el momento de la AA.

\section{RM Cine}

La resonancia magnética $(\mathrm{RM})$ del sueño ha demostrado ser una herramienta útil en la evaluación y planificación quirúrgica de niños con SAHOS persistente ${ }^{36}$. Proporciona imágenes de

\section{Tabla 5. Protocolos DISE \\ Protocolo Cincinnati \\ Inducción con sevoflurano \\ Dexmedetomidina: dosis de carga de $2 \mathrm{mcg} / \mathrm{kg}$ seguido de infusión continua $2 \mathrm{mcg} / \mathrm{kg} / \mathrm{h}$}

\section{Protocolo Antwerp}

Inducción con mezcla de sevoflurano y oxígeno

Propofol: dosis de carga 1-2 mg seguido de infusión continua de $6-10 \mathrm{mg} / \mathrm{kg} / \mathrm{h}$

Ketamina: 1-2 mg/kg administrada al inicio de la dosis de carga de dexmedetomidina

Sin uso de descongestionantes o anestesia tópica

Sin uso de anestesia local nasal o faríngea 
alta resolución de la VAS y sin uso de radiación ionizante siendo particularmente útil en los niños con obstrucción multinivel ${ }^{26}$. Los estudios de RM de sueño incluyen secuencias para evaluar tanto la información anatómica estática como los patrones de colapso dinámico de la VAS. Para ello utilizan la técnica Cine donde las imágenes obtenidas se visualizan secuencialmente a lo largo del ciclo respiratorio ${ }^{37}$. El primer caso clínico donde se aplicó su uso fue en 2001 en el Cincinnati Children's Hospital. Las indicaciones para el estudio de RM Cine incluyen: SAHOS persistente a pesar de cirugía previa (principalmente AA); SAHOS persistente y predisposición a obstrucción multinivel; SAHOS y obesidad severa; y también cuando se anticipa la necesidad de cirugía compleja de VAS para SAHOS, siendo principalmente utilizada en niños con trisomía $21^{36}$.

$\mathrm{Al}$ interpretar los estudios de RM del sueño, se evalúan 2 parámetros principales: el grado de movimiento de las vías respiratorias/patrón de colapso y las anomalías anatómicas. Las posibles anomalías anatómicas incluyen el crecimiento y recurrencia del tejido adenoideo, hiperplasia de amígdalas palatinas, amígdalas linguales prominentes, macroglosia y anormalidades del paladar blando como por ejemplo, en su grosor por infiltración grasa, como ocurre en niños obesos. El colapso intermitente de la rinofaringe o de la vía aérea retrolingual siempre debe considerarse anormal en niños ${ }^{36}$.

Clark y cols. ${ }^{18}$ realizaron un análisis retrospectivo de los registros médicos de niños con SAHOS persistente pos-AA donde compararon los hallazgos de la RM Cine y DISE, encontrando concordancia en el sitio de obstrucción en 10 de los 15 pacientes estudiados, de los cuales 9 correspondieron a un sitio único de obstrucción (67\% DISE vs 73\% RM) siendo principalmente la base de lengua el lugar de colapso de la VAS. Ambas metodologías de estudio requieren sedación y anestésicos similares por lo que su aplicación en conjunto puede entregar mayores herramientas para definir la mejor alternativa terapéutica en pacientes con SAHOS persistente pos-AA. Por ello, Bergeron y cols. ${ }^{38}$ realizaron un estudio prospectivo de casos y controles con el objetivo de evaluar la seguridad del DISE en sala de inducción de RM en comparación a su realización en el quirófano. Evidenciaron ausencia de complica- ciones significativas en ambas situaciones y con tiempos similares en su ejecución por lo cual consideran seguro realizar consecutivamente ambos procedimientos, disminuyendo de esta forma, el número de inducciones anestésicas en este grupo de pacientes y el costo total de estas intervenciones.

\section{Tratamiento del SAHOS persistente}

El tratamiento debe basarse en una evaluación de los factores de riesgo adicionales para la obstrucción de la VAS, la presencia de comorbilidades y una evaluación del patrón y niveles de colapso. Un enfoque de manejo multidisciplinario es altamente deseable con intervenciones de tratamiento médico y/o quirúrgico ${ }^{22}$.

Una vez que él o los sitios de obstrucción persistente de la VAS pos-AA se hayan identificado, se puede proponer un tratamiento individualizado. Estas modalidades de tratamiento complementario: 1. Pueden abordar la obstrucción en regiones específicas de la VAS como, por ejemplo, intervenciones quirúrgicas en base de lengua o tratamiento de ortodoncia; 2. Pueden modificar la permeabilidad de toda la VAS, como es el uso de presión positiva continua de la vía aérea (CPAP) o ventilación no invasiva con presión positiva, o 3. Pueden cambiar las condiciones subyacentes que contribuyen a la enfermedad persistente. Ejemplos de esto último, son un programa de pérdida de peso para la obesidad, tratamiento médico para reducir la inflamación de la VAS o terapia miofuncional para mejorar el tono muscular de la $\mathrm{VAS}^{16}$.

\section{Tratamiento médico}

Actualmente las terapias de manejo médico más comunes para el SAHOS persistente en niños son la baja de peso en pacientes obesos, el uso de CPAP y la indicación de antiinflamatorios de la VAS con montelukast y/o corticoides inhalatorios nasales $(\mathrm{CIN})^{26}$.

\section{Baja de peso}

Uno de los factores de riesgo más claros para SAHOS persistente es la obesidad, que dada la epidemia mundial de malnutrición por exceso es probable que continúe aumentando. Los estudios publicados en población adolescente con obesidad mórbida incluidos en programas residenciales de tratamiento para 
la obesidad o sometidos a cirugía bariátrica muestran efectos beneficiosos en la reducción del IAH. El estudio NANOS, estudio multicéntrico transversal y prospectivo de 117 niños españoles obesos, evidenció una resolución del SAHOS en un $50 \%(\mathrm{IAH}<3)$ en el grupo SAHOS leve sin HAA (edad 10,35 $\pm 2,89$ ) que fueron manejados con modificaciones en la dieta por un tiempo promedio de 11,8 meses $^{39}$. Estos efectos no son necesariamente aplicables a niños más jóvenes con SAHOS y sobrepeso, en quienes la adiposidad contribuye en menor medida a la patogénesis del TRS en comparación con la HAA o las características craneofaciales ${ }^{16}$. De igual manera se recomienda la pérdida de peso como parte del manejo integral del paciente con sobrepeso u obesidad y SAHOS persistente, tanto por sus efectos directos sobre el SAHOS, como también por sus beneficios sobre la salud general ${ }^{26}$.

\section{CPAP}

Si bien la terapia con presión positiva en la vía aérea (PAP) es una alternativa útil para el tratamiento de los niños con SAHOS persistente, existen dos limitantes para su uso ampliado en población pediátrica: la primera son los efectos deletéreos en la anatomía facial por uso prolongado de PAP y la segunda es que lograr una adherencia adecuada puede ser un gran desafío ${ }^{26}$. En un estudio prospectivo multicéntrico que comparó la adherencia y efectividad de la ventilación con CPAP versus PAP binivel en niños con SAHOS, un tercio de los menores ya había abandonado su uso antes de los 6 meses $^{40}$. No hubo diferencia estadística entre CPAP y ventilación PAP binivel, siendo ambas altamente eficaces, pero la adherencia, incluso en un entorno de investigación con seguimiento frecuente, fue subóptima con un uso nocturno promedio de solo $5,3 \pm 2,5$ horas. Otros estudios sugieren que mediante programas de educación y seguimiento de niños en edad escolar y adolescentes es posible lograr una mayor adherencia al uso de PAP y que incluso ante una adherencia subóptima todavía existiría una mejoría significativa en múltiples dominios de la neuroconducta (déficit de atención, somnolencia y comportamiento $)^{41}$.

\section{Montelukast}

Varios estudios han demostrado que la terapia antiinflamatoria con montelukast y/o CIN puede ser beneficiosa en el tratamiento del SAHOS leve debido a la mayor expresión del receptor de leucotrienos y de glucocorticoides en el tejido linfático de los niños con SAHOS ${ }^{42}$. Esto ha llevado a un cambio progresivo en la práctica clínica, por lo que muchos médicos considerarían un tratamiento médico inicial para el SAHOS leve antes de indicar la AA. Sin embargo, en el contexto del SAHOS persistente, la principal preocupación con la terapia médica se ha centrado en su efectividad, ya que la mayor parte del tejido linfoide que presumiblemente es el principal contribuyente al SAHOS se ha eliminado quirúrgicamente ${ }^{26}$. Wang y cols. ${ }^{43}$, evaluaron el efecto del tratamiento con montelukast en 58 niños con SAHOS persistente leve post-AA, observando que el grupo tratado tuvo una mejoría estadísticamente significativa en el IAH (3,55 \pm 1,15 vs $2,20 \pm 0,93 / h$ ), saturación mínima y puntuación del PSQ, mientras que el grupo control sin tratamiento no experimentó cambios significativos durante el estudio.

\section{Tratamiento fonoaudiológico y terapia miofuncional}

Los músculos de la VAS desempeñan un papel fundamental en el mantenimiento de una vía aérea permeable durante el sueño. La respiración bucal se asocia con la posición incorrecta de la lengua, labios hipotónicos y un patrón de deglución alterado lo que puede predisponer a SAHOS persistente pos- $\mathrm{AA}^{16}$.

La terapia miofuncional (TMF) consiste en ejercicios orofaríngeos isotónicos e isométricos repetitivos de la lengua, paladar blando y paredes faríngeas laterales con el objetivo de aumentar el tono muscular en toda la VAS y obtener hábitos respiratorios nasales correctos. Su implementación es más efectiva en niños de 6 años en adelante y está relacionada directamente con el apoyo parental en el cumplimiento de los ejercicios ${ }^{44}$. Villa y cols. ${ }^{45}$ realizaron un ensayo clínico controlado donde evaluaron la eficacia de los ejercicios orofaríngeos en niños mayores de 4 años con SAHOS persistente posAA $(\mathrm{IAH}>1)$. Aleatorizaron a los pacientes $(n=27)$ en 2 grupos y compararon el uso de TMF (más lavados nasales) diariamente durante 2 meses con una frecuencia de al menos 3 sesiones por día en domicilio versus solo 
lavados nasales. La eficacia de los ejercicios orofaríngeos expresados según la variación del IAH pre y postratamiento fue de $58 \%$ para el grupo con TMF y de un 7\% para el grupo que recibió solo lavados nasales. Por lo tanto, la TMF es una alternativa terapéutica o complemento a otras terapias en la recuperación del tono muscular de la VAS en pacientes con SAHOS persistente pos-AA.

\section{Ortodoncia y cirugía maxilofacial}

Las alteraciones ortodóncicas y maxilofaciales pueden ser causa o efecto de una obstrucción persistente de la VAS durante el sueño. Dada su alta prevalencia, es de vital importancia que el otorrinolaringólogo esté alerta e incluya en su examen físico la evaluación de la oclusión dental, paladar duro y anatomía del tercio medio facial y mandibular en pacientes que se presentan a su consulta por ronquidos. Las opciones de tratamiento de ortodoncia para el SAHOS pediátrico incluyen la terapia con aparatos orales y la expansión maxilar rápida (EMR). Los aparatos orales son diseñados para corregir la maloclusión dental del niño y guiar el crecimiento de la lengua y huesos faciales ${ }^{46}$. Una revisión Cochrane encontró que la evidencia disponible es de baja calidad e insuficiente para guiar el tratamiento del SAHOS mediante su uso exclusivo ${ }^{47}$.

\section{Expansión maxilar rápida}

La EMR consiste en distraer la sutura mediopalatina para ampliar el arco maxilar y la apertura piriforme, aumentando el volumen de la VAS en dicho nivel. Un metaanálisis analizó 17 estudios con un total de 314 niños que recibieron EMR como tratamiento del SAHOS con un seguimiento a 3 años y evidenciaron una reducción significativa en el IAH de un $70 \%(8,9 \pm 7,0 / \mathrm{h}$ a $2,7 \pm 3,3 / \mathrm{h})$ y una tasa de curación de un 25,6\% (IAH < 1). Al analizar grupos específicos se observó que el IAH mejoró aún más en niños con AA previa o amígdalas pequeñas (reducción del 73\%-95\%) que en niños con amígdalas grandes (reducción del 61\% $)^{48}$.

\section{Distracción facial}

Otra alternativa terapéutica es la cirugía maxilofacial mediante distracción mediofacial (DMF) o mandibular (DM), las cuales están reservadas para niños con obstrucción importante de la VAS secundarias a anomalías del crecimiento craneofacial o ante fallas al tratamiento conservador en lactantes con hipoplasia mandibular ${ }^{46}$. Un metaanálisis encontró que el 91,3\% de los recién nacidos y lactantes pudieron evitar la intubación o la traqueotomía después de la osteogénesis por DM y aunque los datos polisomnográficos posoperatorios son limitados, esta cirugía se ha asociado con una reducción significativa del $\mathrm{IAH}^{49}$. Niños con alteraciones significativas del crecimiento maxilar también pueden beneficiarse de la cirugía esquelética facial. Múltiples estudios han demostrado una asociación entre la DMF y las mejoras en el volumen de la VAS y el SAHOS.

\section{Cirugía de la vía aérea superior}

Un nuevo crecimiento adenoideo o la identificación de la obstrucción de la VAS fuera de las amígdalas palatinas y adenoides en niños con SAHOS persistente pos-AA pueden ser una indicación para intervenciones quirúrgicas adicionales desde el punto de vista otorrinolaringológico. La hipertrofia de las amígdalas linguales (HAL) y la laringomalacia de inicio tardío se han descrito como las dos causas más comunes de obstrucción de la VAS en niños con SAHOS residual ${ }^{16}$. Las posibles intervenciones quirúrgicas incluyen: adenoidectomía para aquellos con recrecimiento de adenoides, septoplastía y/o turbinoplastía, cirugía palatofaríngea, amigdalectomía lingual (AL), cirugía de base de lengua, estimulación del nervio hipogloso y supraglotoplastía.

\section{Cirugía nasal y palatofaríngea}

Alternativas para el manejo de la obstrucción de la VAS a nivel nasal son la septoplastía en casos particulares y la turbinoplastía endoscópica en casos de hipertrofia de cornetes inferiores mediante uso de microdebridador o radiofrecuencia. Ambas técnicas se deben realizar de forma conservadora, respetando cuidadosamente la integridad de la mucosa nasoseptal y minimizando el daño sobre el cartílago septal. La cirugía de cornetes inferiores ha demostrado beneficios adicionales en términos de IAH y calidad de vida al asociarse a AA junto con una mejoría significativa y a largo plazo en la obstrucción nasal. La septoplastía 
puede mejorar la tolerancia a CPAP en niños mayores $^{50}$.

Respecto a la cirugía palatofaríngea: aunque la uvulopalatofaringoplastía, ha demostrado mejoras en el IAH y parámetros PSG, no está recomendada en niños por la alta tasa de enfermedad persistente, siendo reemplazada por procedimientos palatales desarrollados para abordar patrones específicos de colapso guiados por DISE. Una de ellas es la faringoplastía de expansión donde un estudio demostró disminución del IAH en pacientes con SAHOS grave asociado a amigdalectomía ${ }^{51}$. Sin embargo, la literatura es escasa respecto a su utilidad en niños con SAHOS persistente y no está exenta de riesgos como insuficiencia velofaríngea o estenosis nasofaríngea por lo que no constituye una técnica ampliamente recomendada ${ }^{50}$.

\section{Obstrucción a nivel de base de la lengua}

Es la base de la lengua el principal sitio de obstrucción de VAS en niños con SAHOS persistente. Esta obstrucción está dada por diversas patologías, siendo las más frecuentes la hipertrofia de las amígdalas linguales, la glosoptosis y la macroglosia. Las opciones quirúrgicas para abordar la obstrucción a este nivel incluyen la AL, la glosectomía posterior de línea media, suspensión de lengua, suspensión del hioides y estimulación del nervio hipogloso. De estos, la AL es el procedimiento de lengua más frecuentemente efectuado en niños con SAHOS persistente ${ }^{50,52}$. A continuación, discutiremos algunas de ellas y en la Figura 1 proponemos un algoritmo de manejo para la obstrucción a este nivel.

\section{Amigdalectomía lingual}

Convencionalmente, el tamaño de la amígdala lingual se evalúa con estudios de imagen, como la TC o la RM y, actualmente, mediante el uso de DISE. Una vez confirmada la obstrucción respiratoria por esta causa está indicada la AL. Kang y cols. ${ }^{52}$ realizaron un metaanálisis con el objetivo de evaluar los cambios

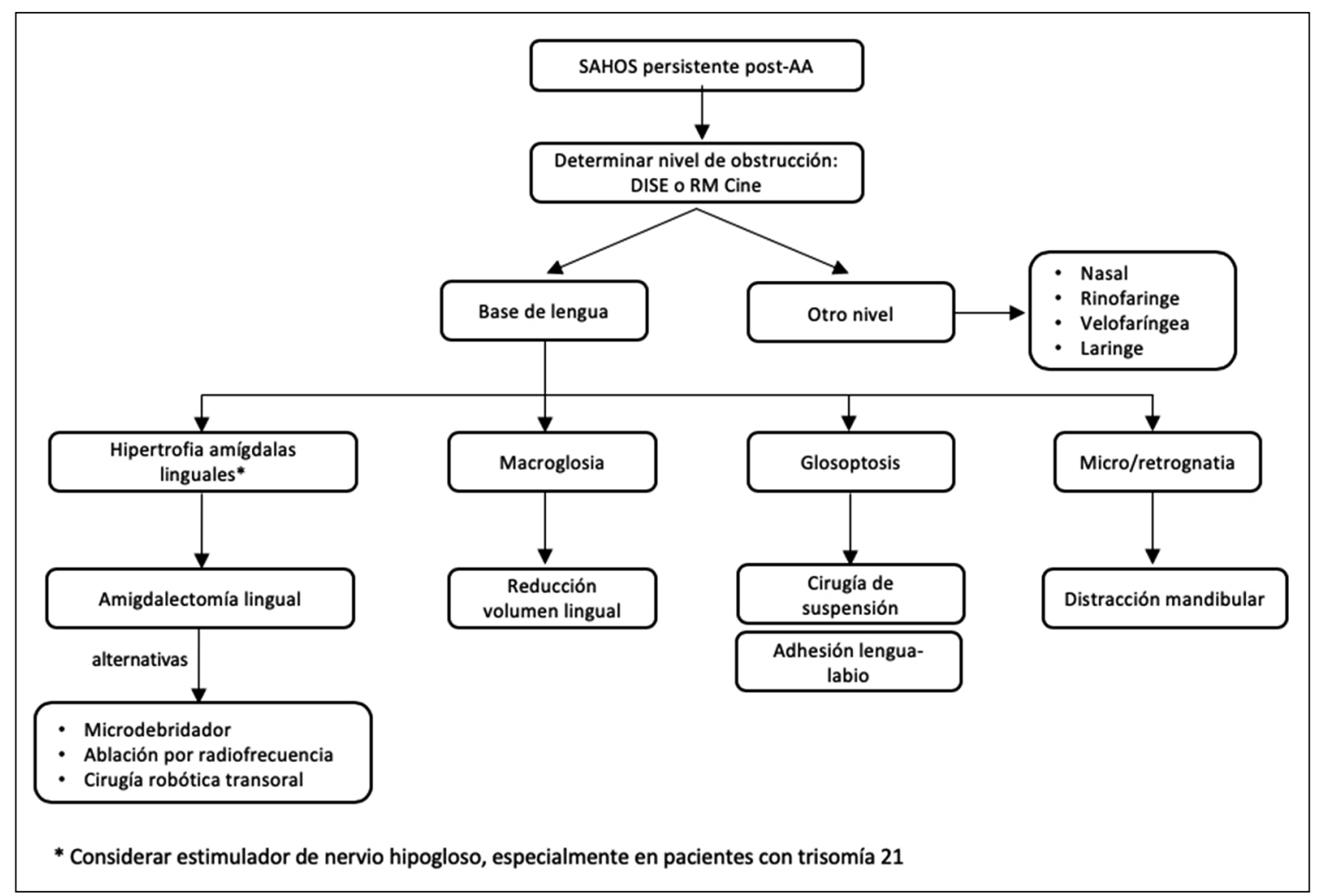

Figura 1. Manejo de obstrucción base de lengua. 
en el IAH y la saturación mínima de oxígeno después de la AL junto con la tasa de éxito del procedimiento para el tratamiento del SAHOS en niños. Analizaron 4 estudios con un total de 73 pacientes con promedio de edad de 8,3 años. Concluyeron que la AL mejora en un promedio de 8,9 el IAH y $6 \%$ la saturación mínima. La tasa de éxito fue de $17 \%$ para $\mathrm{IAH}<1$ y de $51 \%$ para $\mathrm{IAH}<5 \mathrm{y}$, por lo tanto, consideran la $\mathrm{AL}$ como un tratamiento quirúrgico eficaz para niños con SAHOS causado por la HAL ${ }^{52}$. Existen diversas técnicas quirúrgicas para extirpar las amígdalas linguales en niños, estas incluyen el uso de microdebridador, ablación con radiofrecuencia, láser de $\mathrm{CO}_{2}$ o cirugía robótica transoral. Las principales complicaciones de la AL son sangrado intra y posoperatorio, depresión respiratoria por obstrucción de la VAS secundario al edema de la base de lengua y neumonía. Ante complicaciones respiratorias, el manejo con oxigenoterapia y corticoides es efectivo en la mayoría de los casos siendo la intubación orotraqueal una medida excepcional. Es importante señalar que estos pacientes requieren monitorización y cuidados postoperatorios estrictos para el manejo oportuno de eventuales complicaciones ${ }^{50,52}$.

\section{Estimulación del nervio hipogloso}

El SAHOS afecta hasta en un $80 \%$ a los pacientes con trisomía 21 y un $60 \%$ de ellos evolucionará con SAHOS persistente pos-AA debido principalmente a la reducción del tono muscular, macroglosia, hipoplasia maxilar e $\mathrm{HAL}^{53}$. Los tratamientos no invasivos en estos pacientes, si bien son efectivos, presentan altas tasas de no adherencia y abandono (40\% a $50 \%$ ) y por su parte, los tratamientos quirúrgicos no están exentos de riesgos y complicaciones perioperatorias. Debido a lo anterior es que Diercks y cols. ${ }^{53}$, desarrollaron un estudio piloto para evaluar principalmente la seguridad del uso de estimuladores de nervio hipogloso en niños. Estos dispositivos implantables consisten en un electrodo sensor colocado entre los músculos intercostales y un cable de estimulación instalado alrededor de las ramas anteriores del nervio hipogloso que suministra impulsos eléctricos a los músculos protrusores de la lengua en el momento de la inspiración, aliviando la obstrucción de la VAS durante el sueño. Estos dispositivos han demostrado ser un tratamiento eficaz y bien tolerado en determinados grupos de adultos con SAHOS. En una publicación reciente, Jayawardena y cols. ${ }^{54}$ analizaron retrospectivamente su experiencia en 23 niños con trisomía 21 en quienes introdujeron modificaciones en la técnica de implantación respecto a los adultos. Ninguno de los pacientes presentó complicaciones mayores cardiopulmonares y concluyeron que los cambios realizados mejoran tanto la seguridad como la eficacia del procedimiento en la población pediátrica.

\section{Supraglotoplastía}

Existe un subconjunto de niños con SAHOS que tienen laringomalacia donde el colapso de las estructuras supraglóticas puede generar estridor inspiratorio, el cual es desencadenado por factores como infecciones de la VAS, alimentación, agitación y posición supina, pero también, existe un grupo menor de este subgrupo de pacientes que presentan colapso supraglótico solo durante el sueño, cuadro conocido como laringomalacia exclusiva del sueño (LMES) o laringomalacia oculta. La edad de presentación es mayor que la de pacientes con laringomalacia congénita, fluctuando entre los 2 y 18 años, con una incidencia estimada de $3,9 \%$ y pudiendo ser diagnosticados mediante DISE. Diversos estudios han informado los beneficios de la supraglotoplastía (SGP) en el SAHOS secundario a LMES, llegando incluso a la curación. Camacho y cols. ${ }^{55}$ realizaron un metaanálisis de la literatura internacional y seleccionaron 13 estudios con un total de 138 niños entre 1 mes y 12,6 años de edad, de los cuales 64 pacientes tenían laringomalacia exclusiva del sueño y el $77,4 \%$ había fallado a la AA. Evidenciaron una reducción del $76,4 \%$ en el IAH $(14,0 \pm 16,5$ a $3,3 \pm 4,0)$, una mejoría significativa en la saturación mínima y una tasa de curación (IAH $<1$ ) de 10,5\%. Otros estudios informan mayores tasas de curación global del SAHOS en pacientes con laringomalacia (congénita y oculta) alcanzando valores entre un $58 \%$ y $72 \%$ al considerar niños tratados en forma primaria con SGP o bien, posterior a la AA, pero usando valores más flexibles de IAH $(<5)$ como criterio de resolución del SAHOS $^{56}$. En conclusión, la supraglotoplastía es un procedimiento útil para el manejo del 


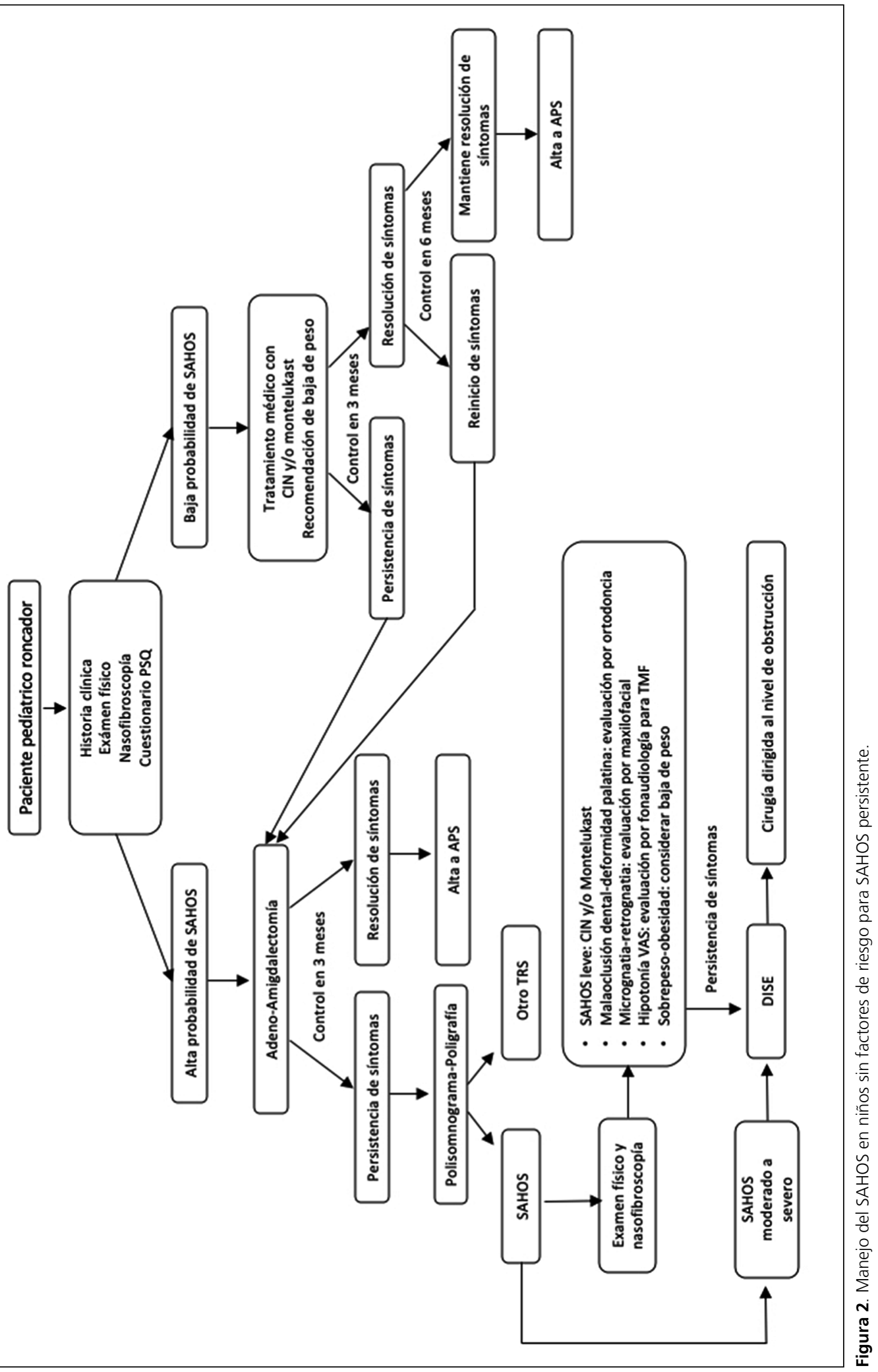


SAHOS persistente en un grupo especial de pacientes con laringomalacia.

\section{Conclusión}

La alta prevalencia de TRS en población infantil chilena sumado a la creciente malnutrición por exceso y las consecuencias mórbidas en el ámbito neuroconductual del SAHOS convierten a esta patología en un problema de salud grave y preocupante que impacta negativamente en el desarrollo normal de nuestros niños. Es prioritario realizar un estudio y manejo adecuados y oportunos para contrarrestar los efectos deletéreos de este síndrome en la población pediátrica. Existen múltiples causas de SAHOS y si bien la HAA es la principal, su resección no asegura la resolución del SAHOS en un importante grupo de pacientes, por lo cual es indispensable el seguimiento de los niños sometidos a AA, sobre todo a aquellos con factores de riesgo para SAHOS persistente o en quienes se mantienen sintomáticos pos-AA. A la fecha no existe consenso respecto a la definición polisomnográfica del SAHOS residual, pero lo que sí está claro, es que este grupo de pacientes deben ser estudiados acabadamente mediante DISE o RM Cine para identificar el o los niveles de obstrucción de la VAS, siendo la base de lengua el lugar más frecuente. Las alternativas de tratamiento para el SAHOS persistente son múltiples y abarcan terapias de manejo médico y quirúrgico como la EMR, distracción facial y cirugías de la vía aérea. Las decisiones terapéuticas idóneas para el SAHOS persistente pos-AA deben ser analizadas en forma multidisciplinaria e individualizada para cada paciente y es importante que estemos presentes en dichos equipos, ya que como otorrinolaringólogos tenemos herramientas indispensables para el adecuado abordaje de estos niños. De acuerdo a nuestra revisión de la literatura, proponemos el siguiente manejo de pacientes con sospecha de SAHOS considerando los recursos limitados en nuestro sistema de salud (Figura 2).

\section{Bibliografía}

1. Zenteno D, Verbal D, Barraza C, Fuentes C.
Epidemiología de los trastornos respiratorios del sueño en pediatría. Neumol Pediatr. 2017; 12(2):4954.

2. Kaditis AG, Alonso Álvarez ML, Boudewyns A, et al. Obstructive sleep disordered breathing in 2- to 18-year-old children: diagnosis and management. Eur Respir J. 2016;47(1):69-94. doi: 10.1183/13993003.00385-2015

3. Lloberes P, Durán-cantolla J, Martínezgarcía M, et al. Diagnóstico y tratamiento del síndrome de apneas-hipopneas del sueño. Arch Bronconeumol 2011;47(3):143-156. doi: 10.1016/j. arbres.2011.01.001.

4. Paiva T. Epidemiology of Sleep Disorders in Children and Adolescents. Sleep Disorders in Children. 2017;5367. doi: 10.1007/978-3-319-28640-2.

5. Sánchez T, Rojas C, Casals M, et al. Trastornos respiratorios del sueño en niños escolares chilenos: prevalencia y factores de riesgo. Rev Chil Pediatr. 2018;89:718-725. doi: 10.4067/S037041062018005000902.

6. Chervin RD, Hedger K, Dillon JE, Pituch KJ. Pediatric sleep questionnaire (PSQ): Validity and reliability of scales for sleep-disordered breathing, snoring, sleepiness, and behavioral problems. Sleep Med. 2000;1(1):21-32. doi: 10.1016/S13899457(99)00009-X.

7. Elso M, Brockmann P, Zenteno D. Consecuencias del síndrome de apnea obstructiva del sueño. Rev Chil Pediatr. 2013; 84 (2):128-137.

8. Ehsan Z, Ishman S. Pediatric obstructive sleep apnea. Otolaryngol Clin N Am. 2016;49:1449-64. doi: 10.1016/j.otc.2016.07.001.

9. Ishman SL. The Role of Sleep Studies in Children Who Snore. JAMA Otolaryngol Head Neck Surg. 2016;142(2):179-181. doi: 10.1001/jamaoto.2015.1.

10. Marcus C, Chapman D, Ward S, et al. Clinical practice guideline: Diagnosis and management of childhood obstructive sleep apnea syndrome. Pediatrics. 2002;109(4):704-712. doi: 10.1542/ peds.109.4.704.

11. Alonso M, Terán J, Cordero J, et al. Fiabilidad de la poligrafía respiratoria para el diagnóstico del síndrome de apneas-hipopneas durante el sueño en niños. Arch Bronconeumol. 2008;44(6):318-323. doi: $10.1157 / 13123089$.

12. Tan H-L, Gozal D, Ramírez H, Bandla H, Kheirandish-Gozal L. Overnight polysomnography versus respiratory polygraphy in the diagnosis of pediatric obstructive sleep apnea. Sleep. 2014;37(2):255-260. doi: 10.5665/sleep.3392.

13. Brouillette R, Morielli A, Leimanis A, Waters K, Luciano R, Ducharme F. Nocturnal pulse oximetry as an abbreviated testing modality for pediatricobstructive sleep apnea. Pediatrics. 2000;105(2):405-412. doi: 10.1542/peds.105.2.405.

14. Nixon G, Kermack A, Davis G, et al. Planning 
adenotonsillectomy in children with obstructive sleep apnea: The Role of Overnight Oximetry. Pediatrics. 2004;113(1):e19-e25. doi: 10.1542/peds.113.1.e19.

15. Bhattacharjee R, Kheirandish-Gozal L, Spruyt K, et al. Adenotonsillectomy outcomes in treatment of obstructive sleep apnea in children: A multicenter retrospective study. Am J Respir Crit Care Med. 2010;182(5):676-683. doi: 10.1164/rccm.2009121930OC.

16. Boudewyns A, Abel F, Alexopoulos E, et al. Adenotonsillectomy to Treat Obstructive Sleep Apnea : Is It Enough? Pediatr Pulmonol. 2017;52:699709. doi: 10.1002/ppul.23641.

17. Mitchell RB, Archer SM, Ishman SL, et al. Clinical Practice Guideline: Tonsillectomy in Children (Update). Otolaryngol Head Neck Surg. 2019;160(1_ suppl):S1-S42. doi: 10.1177/0194599818801757.

18. Clark C, Ulualp S. Multimodality assessment of upper airway obstruction in children with persistent obstructive sleep apnea after adenotonsillectomy. Laryngoscope. 2017;127:1224-1230. doi: 10.1002/ lary.26174.

19. Tunkel DE. Polysomnography before tonsillectomy in children: who and when? Otolaryngol Head Neck Surg. 2012;146(2):191-195. doi: 10.1177/0194599811429236.

20. Friedman M, Wilson M, Lin HC, Chang HW. Updated systematic review of tonsillectomy and adenoidectomy for treatment of pediatric obstructive sleep apnea/hypopnea syndrome. Otolaryngol Head Neck Surg. 2009;140(6):800-808. doi: 10.1016/j. otohns.2009.01.043

21. Costa DJ, Mitchell R. Adenotonsillectomy for obstructive sleep apnea in obese children: a meta-analysis. Otolaryngol Head Neck Surg. 2009;140(4):455-460. doi: 10.1016/j. otohns.2008.12.038.

22. Imanguli M, Ulualp S. Risk factors for residual obstructive sleep apnea after adenotonsillectomy in children. Laryngoscope. 2016;126(11):2624-2629. doi: 10.1002/lary.25979.

23. Wise M, Nichols C, Grigg-Damberger M, et al. Executive summary of respiratory indications for polysomnography in children: An evidence-based review. Sleep. 2011;34(3):389-98. doi: 10.1093/ sleep/34.3.389.

24. Roland PS, Rosenfeld RM, Brooks LJ, et al. Clinical practice guideline: Polysomnography for sleepdisordered breathing prior to tonsillectomy in children. Otolaryngol Head Neck Surg. 2011;145(1 Suppl):S1-S15. doi: 10.1177/0194599811409837.

25. Marcus C, Brooks L, Draper K, et al. Clinical Practice Guideline: Diagnosis and management of childhood obstructive sleep apnea syndrome. Pediatrics. 2012;130:576-584. doi: 10.1542/peds.2012-1671.

26. Tan HL, Kheirandish-Gozal L, Gozal D. Obstructive sleep apnea in children: update on the recognition, treatment and management of persistent disease. Expert Rev Respir Med. 2016;10(4):431-439. doi: 10.1586/17476348.2016.1163224.

27. Gozal D, Jortani S, Snow AB, et al. Two-dimensional differential in-gel electrophoresis proteomic approaches reveal urine candidate biomarkers in pediatric obstructive sleep apnea. Am J Respir Crit Care Med. 2009;180(12):1253-1261. doi: 10.1164/ rccm.200905-0765OC.

28. Pabla L, Duffin J, Flood L, Blackmore K. Paediatric obstructive sleep apnoea: is our operative management evidence-based? J Laryngol Otol. 2018;132(4):293-298. doi: 10.1017/ S002221511800021X.

29. Croft C, Pringle M. Sleep nasendoscopy: a technique of assessment in snoring and obstructive sleep apnoea. Clin Otolaryngol Allied Sci. 1991;16(5):504509. doi: 10.1111/j.1365-2273.1991.tb01050.x.

30. He S, Peddireddy NS, Smith DF, et al. Outcomes of Drug-Induced Sleep Endoscopy-Directed Surgery for Pediatric Obstructive Sleep Apnea. Otolaryngol Head Neck Surg. 2018;158(3):559-565. doi: 10.1177/0194599817740332

31. Boudewyns A, Saldien V, Van de Heyning P, Verhulst $S$. Drug-induced sedation endoscopy in surgically naïve infants and children with obstructive sleep apnea: impact on treatment decision and outcome. Sleep Breath. 2018;22(2):503-510. doi: 10.1007/ s11325-017-1581-7.

32. Traxdorf M, Tschaikowsky K, Scherl C, Bauer J, Iro H, Angerer F. Drug-Induced Sleep Endoscopy (DISE) with Target Controlled Infusion (TCI) and Bispectral Analysis in Obstructive Sleep Apnea. J Vis Exp. 2016;(118):54739. doi: 10.3791/54739.

33. Flores J, Villanueva S, Macías H, Sánchez E, Lugo R. Dexmedetomidina en la evaluación endoscópica de sueño inducido en apnea obstructiva del sueño. $A n$ Orl Mex. 2016; 61(3):190-197.

34. Charakorn N, Kezirian EJ. Drug-Induced Sleep Endoscopy. Otolaryngol Clin North Am. 2016;49(6):1359-1372. doi: 10.1016/j.otc.2016.06.002.

35. Friedman N, Parikh S, Ishman S, et al. The current state of pediatric drug-induced sleep endoscopy. Laryngoscope. 2017;127(1):266-272. doi: 10.1002/ lary.26091.

36. Donnelly L. Magnetic resonance sleep studies in the evaluation of children with obstructive sleep apnea. Semin Ultrasound CT MR. 2010;31(2):107-115. doi: 10.1053/j.sult.2009.12.001.

37. Donnelly LF. Obstructive sleep apnea in pediatric patients: evaluation with cine MR sleep studies. Radiology. 2005;236(3):768-778. doi: 10.1148/ radiol.2363040306.

38. Bergeron M, Duggins A, Chini B, Ishman SL. Clinical outcomes after shared decision-making tools with families of children with obstructive sleep apnea without tonsillar hypertrophy. Laryngoscope. 
2019;129(11):2646-2651. doi: 10.1002/lary.27653.

39. Alonso-Álvarez M, Terán-Santos J, Navazo-Egüia A, et al. Treatment outcomes of obstructive sleep apnoea in obese community-dwelling children: the NANOS study. Eur Respir J.2015;46(3):717-727. doi: $10.1183 / 09031936.00013815$.

40. Marcus C, Rosen G, Davidson S, et al. Adherence to and effectiveness of positive airway pressure therapy in children with obstructive sleep apnea. Pediatrics. 2006;117(3): e442-e451. doi: 10.1542/peds.2005-1634.

41. Uong E, Epperson M, Bathon S, Jeffe D. Adherence to nasal positive airway pressure therapy among schoolaged children and adolescents with obstructive sleep apnea syndrome. Pediatrics. 2007;120(5):1203-11. doi: 10.1542/peds.2006-2731.

42. Kheirandish-Gozal L, Bandla H, Gozal D. Montelukast for Children with Obstructive Sleep Apnea: Results of a Double-Blind, Randomized, Placebo-Controlled Trial. Ann Am Thorac Soc. 2016;13(10):1736-41. doi: 10.1513/AnnalsATS.201606-432OC.

43. Wang B, Liang J. The Effect of Montelukast on Mild Persistent OSA after Adenotonsillectomy in Children: A Preliminary Study. Otolaryngol Head Neck Surg. 2017;156(5):952-954. doi: 10.1177/0194599817696501.

44. Guilleminault C, Huang Y, Monteyrol PJ, Sato $\mathrm{R}$, Quo S, Lin CH. Critical role of myofascial reeducation in pediatric sleep-disordered breathing. Sleep Med. 2013;14(6):518-525. doi: 10.1016/j. sleep.2013.01.013.

45. Villa M, Brasili L, Ferretti A, et al. Oropharyngeal exercises to reduce symptoms of OSA after AT. Sleep Breath. 2015;19(1):281-289. doi: 10.1007/s11325-0141011-z.

46. Garg R, Afifi A, Garland C, Sánchez R, Mount D. Pediatric Obstructive Sleep Apnea: Consensus, Controversy, and Craniofacial Considerations. Plast Reconstr Surg. 2017;140(5):987-997. doi: 10.1097/ PRS.0000000000003752.

47. Carvalho F, Lentini-Oliveira D, Prado L, Prado $\mathrm{G}$, Carvalho L. Oral appliances and functional orthopaedic appliances for obstructive sleep apnoea in children. Cochrane Database Syst Rev.
2016;10(10):CD005520. doi: 10.1002/14651858. CD005520.pub3.

48. Camacho M, Chang E, Song S, et al. Rapid maxillary expansion for pediatric obstructive sleep apnea: A systematic review and meta-analysis. Laryngoscope. 2017;127(7):1712-9. doi: 10.1002/lary.26352.

49. Ow A, Cheung L. Meta-analysis of mandibular distraction osteogenesis: clinical applications and functional outcomes. Plast Reconstr Surg. 2008;121(3):54e-69e. doi: 10.1097/01. prs.0000299285.97379.35.

50. Bluher A, Ishman S, Baldassari C. Managing the Child with Persistent Sleep Apnea. Otolaryngol Clin North Am. 2019;52(5):891-901. doi: 10.1016/j. otc.2019.06.004.

51. Ulualp SO. Modified expansion sphincter pharyngoplasty for treatment of children with obstructive sleep apnea. JAMA Otolaryngol Head Neck Surg. 2014;140(9):817-822. doi: 10.1001/ jamaoto.2014.1329.

52. Kang KT, Koltai PJ, Lee CH, Lin MT, Hsu WC. Lingual Tonsillectomy for Treatment of Pediatric Obstructive Sleep Apnea: A Meta-analysis. JAMA Otolaryngol Head Neck Surg. 2017;143(6):561-568. doi: 10.1001/jamaoto.2016.4274.

53. Diercks GR, Wentland C, Keamy D, et al. Hypoglossal Nerve Stimulation in Adolescents With Down Syndrome and Obstructive Sleep Apnea. JAMA Otolaryngol Head Neck Surg. 2018;144(1):37-42. doi: 10.1001/jamaoto.2017.1871.

54. Jayawardena A, Randolph G, Hartnick C. Pediatric Modifications to Hypoglossal Nerve Stimulation for Obstructive Sleep Apnea: How I Do It. Laryngoscope. 2020; 1-2. doi: 10.1002/lary.28661.

55. Camacho M, Dunn B, Torre C, et al. Supraglottoplasty for laryngomalacia with obstructive sleep apnea: A systematic review and meta-analysis. Laryngoscope. 2016;126(5):1246-1255. doi: 10.1002/ lary.25827.

56. Manickam P, Shott S, Boss E, et al. Systematic review of site of obstruction identification and non-CPAP treatment options for children with persistent pediatric obstructive sleep apnea. Laryngoscope. 2016;126(2):491-500. doi: 10.1002/lary.25459. 